\title{
Estudo fitoquímico de Davilla rugosa: flavonóides e terpenóides
}

\author{
Jorge M. David ${ }^{*}$, Jurema C. Souza ${ }^{1}$, Maria L. Silva Guedes², Juceni P. David ${ }^{3}$ \\ ${ }^{1}$ Instituto de Química, Universidade Federal da Bahia, Campus Ondina, 40170-290, \\ Salvador, BA, Brasil, \\ ${ }^{2}$ Faculdade de Farmácia, Universidade Federal da Bahia, Campus Ondina, 40170-290, \\ Salvador, BA, Brasil, \\ ${ }^{3}$ Instituto de Biologia, Universidade Federal da Bahia, Campus Ondina, 40170-290, \\ Salvador, BA, Brasil
}

\begin{abstract}
RESUMO: Este trabalho descreve o isolamento e caracterização de alguns metabólitos presentes nos caules de Davilla rugosa (Dilleniaceae), conhecida vulgarmente como cipó-caboclo e utilizada pela população na medicina tradicional brasileira para diversas enfermidades. $\mathrm{O}$ extrato hidrometanólico foi particionado entre hexano, clorofórmio e acetato de etila. Da fase hexânica foram obtidos a friedelina, sitostenona e ácido betulínico. Da fase clorofórmica foram isolados através de técnicas cromatográficas o ácido betulínico e a narigenina. Enquanto que do extrato acetato de etila foram obtidos dois outros flavonóides, quercetina e 4'-O-metil taxifolina. As substâncias tiveram suas estruturas elucidadas através da análise de dados no UV e de RMN.
\end{abstract}

Unitermos: Davilla rugosa, Dilleniaceae, ácido betulínico, flavonóides.

\begin{abstract}
Phytochemical study of Davilla rugosa: flavonoids and terpenoids". This work describes the first chemical study of the Brazilian medicinal plant Davilla rugosa (Dilleniaceae) popularly known as "cipó-caboclo". The hydromethanolic extract of the stems was partitioned between hexane, $\mathrm{CHCl}_{3}$ and EtOAc. Chromagraphic methods of fractions resulted in isolation of friedelin, sitostenone, betulinic acid, narigenin, quercetin and 4'-O-methyl taxifolin. The compounds were identified by spectrometric data analyses.
\end{abstract}

Keywords: Davilla rugosa, Dilleniaceae, betulinic acid, flavonoids.

\section{INTRODUÇÃO}

A família Dilleniaceae é formada de aproximadamente 300 espécies, exclusivamente tropicais, sendo na sua maioria compreendida de cipós e arbustos. No Brasil, ocorrem espécies dos gêneros Davilla, Curatella, Tetracera e Doliocarpus, distribuídas nos cerrados e em todo o Nordeste (Schultz, 1984). $\mathrm{Na}$ Bahia, algumas espécies desta família são nativas e encontram aplicação como plantas medicinais pela população. Entretanto, estudos fitoquímicos na literatura, com espécies brasileiras ainda são raros e realizados de forma parcial.

Davilla rugosa Poir. et St. Hill, espécie de origem brasileira, é conhecida popularmente como cipócaboclo e uma espécie de origem brasileira, amplamente utilizada pela população brasileira no tratamento de diferentes distúrbios, tais como, elefantíase, inchaço dos membros, diurético, afrodisíaco e úlcera (Coimbra, 1942; Corrêa, 1984). A atividade anti-úlcera de frações do extrato hidroalcoólico desta espécie foi anteriormente avaliada em cobaias (Bacchi, 1986; Guaraldo et al., 2001). Este estudo demonstrou que o extrato apresentou atividade de proteção para desenvolvimento de úlceras gástricas e, que as substâncias ativas estavam nas frações mais polares. Além disso, as mesmas frações testadas também sugeriram aumento na atividade motora (efeito estimulante) quando estudado nos mesmos modelos (Guaraldo et al., 2000). Não obstante as atividades demonstradas e da grande utilização popular da espécie, até o momento a mesma ainda não havia sido estudada do ponto de vista químico. Anteriormente a literatura registra o isolamento de flavonóides de D. flexuosa (David et al., 1996) e o estudo farmacobotânico (Soares et al., 2005) antimicrobiano (Michelin et al., 2005) e farmacológico (Carlos et al., 2005) de Davilla elliptica. Neste trabalho relata-se pela primeira vez o isolamento e identificação de flavonóides e terpenos dos extratos orgânicos dos caules de D. rugosa.

\section{MATERIAL E MÉTODOS}

O espécime estudado foi coletado nos arredores da Lagoa do Abaeté do Parque Metropolitano do Abaeté, Salvador, BA, identificado e uma excicata encontra-se depositada no Herbário Alexandre Leal Costa, do Instituto de Biologia da Universidade Federal da Bahia.

Os espectros no Ultravioleta foram registrados em espectrofotômetro da Varian mod. CARY I enquanto 
que os espectros de RMN foram registrados em espectrômetro Gemini 300 (Varian). Os espectros foram registrados utilizando-se solventes deuterados e os sinais foram corrigidos com o valor dos solventes em relação ao TMS.

Os caules de $D$. rugosa coletados foram secos em estufa com ventilação e posteriormente moídos (403 g). Em seguida foram submetidos a maceração com $\mathrm{MeOH}$ a temperatura ambiente por 48 horas, procedimento este repetido por duas vezes consecutivas. No extrato obtido foi adicionado água e particionado entre hexano, clorofórmio fornecendo assim as respectivas fases; hexânica $(2,16$ g) e $\mathrm{CHCl}_{3}(1,82 \mathrm{~g})$. $\mathrm{O}$ extrato hidrometanólico foi concentrado de modo a eliminar o metanol e este foi submetido novamente a partição seqüencial com acetato de etila e butanol, fornecendo assim as fases AcOEt $(1,36$ g) e $\mathrm{BuOH}(8,90 \mathrm{~g})$.

A fração hexânica foi submetida a coluna cromatográfica sob gel de sílica 60 e eluída com misturas de hexano:AcOEt em diferentes proporções. Desta coluna foram obtidas 50 frações de $30 \mathrm{~mL}$ cada. Das frações eluídas com hexano:AcOEt (95:5) foram obtidos 50,3 $\mathrm{mg}$ de friedelina. As frações eluídas com $10 \%$ de acetato de etila foram submetidas a CCDC em gel de sílica 60 e as placas foram eluídas com mistura de benzeno:AcOEt (92:8), sendo desta maneira isolado a sitostenona (16,2 $\mathrm{mg})$. Já das frações eluídas com hexano:AcOEt (4:1) foi obtido um sólido que foi identificado como ácido betulínico $(1,19 \mathrm{~g})$.

A fração $\mathrm{CHCl}_{3}$ foi submetida a coluna cromatográfica sob gel de sílica 60 e eluída com misturas de benzeno e AcOEt em diferentes proporções obtendose 16 frações de $50 \mathrm{~mL}$ cada. As frações eluídas com $40 \%$ de AcOEt (1,60 g) foram recristalizadas com metanol obtendo-se desta maneira $1,30 \mathrm{~g}$ de ácido betulínico (1) e 25,2 mg de narigenina (2) na água mãe. Paralelamente, a fração AcOEt foi submetido a coluna cromatográfica sob gel de sílica 60 e eluída com mistura de $\mathrm{CHCl}_{3}: \mathrm{MeOH}$

Tabela 1. Dados de RMN de ${ }^{1} \mathrm{H}$ e de ${ }^{13} \mathrm{C}\left[300\right.$ e $75 \mathrm{MHz}$, acetona-d $\left.{ }_{6}, J(\mathrm{~Hz}), \delta(\mathrm{ppm})\right]$ dos flavonóides de $D$. rugosa

\begin{tabular}{|c|c|c|c|c|c|}
\hline \multirow[t]{2}{*}{ Posição } & \multicolumn{3}{|c|}{$\delta_{\mathrm{H}}$} & \multicolumn{2}{|c|}{$\delta_{\mathrm{C}}$} \\
\hline & 2 & 3 & 4 & 2 & 4 \\
\hline 2 & $\begin{array}{c}5,50 d d \\
(12,9 ; 2,4)\end{array}$ & - & $\begin{array}{l}5,03 d \\
(10,0)\end{array}$ & 79,68 & 85,05 \\
\hline 3 & $\begin{array}{c}2,87 d d / 3,27 d d \\
(17,0 ; 12,9) /(17,1 ; 2,4)\end{array}$ & - & $\begin{array}{l}4,64 d \\
(10,0)\end{array}$ & 43,38 & 73,71 \\
\hline 4 & - & - & - & 196,48 & 195,0 \\
\hline 5 & - & - & - & 165,23 & $164,5^{*}$ \\
\hline 6 & $\begin{array}{c}6,47 d \\
(1,8)\end{array}$ & $\begin{array}{c}6,45 d \\
(2,0)\end{array}$ & $\begin{array}{c}6,06 d \\
(2,1)\end{array}$ & 97,29 & 95,15 \\
\hline 7 & - & - & - & 168,50 & $168,2^{*}$ \\
\hline 8 & $\begin{array}{c}6,37 d \\
(1,8)\end{array}$ & $\begin{array}{c}6,23 d \\
(2,0)\end{array}$ & $\begin{array}{c}6,03 d \\
(2,1)\end{array}$ & 96,19 & 96,21 \\
\hline 9 & - & - & - & 164,08 & $164,6^{*}$ \\
\hline 10 & - & - & - & 102,96 & 107,8 \\
\hline $1^{\prime}$ & & - & - & 129,85 & 130,5 \\
\hline $2^{\prime}$ & $\begin{array}{c}7,56 d \\
(8,5)\end{array}$ & $\begin{array}{c}7,80 d \\
(1,8)\end{array}$ & $\begin{array}{c}7,21 d \\
(1,9)\end{array}$ & 128,81 & 116,34 \\
\hline $3^{\prime}$ & $\begin{array}{c}6,96 d \\
(8,5)\end{array}$ & - & - & 116,48 & 146,20 \\
\hline $4^{\prime}$ & & - & - & 156,4 & 147,80 \\
\hline $5^{\prime}$ & $\begin{array}{c}6,96 d \\
(8,5)\end{array}$ & $\begin{array}{c}7,00 d \\
(7,8)\end{array}$ & $6,70-6,90 \mathrm{~m}$ & 128,81 & 116,24 \\
\hline $6^{\prime}$ & $\begin{array}{c}7,56 d \\
(8,5)\end{array}$ & $\begin{array}{c}7,70 d d \\
(1,8 ; 7,8)\end{array}$ & $6,70-6,90 \mathrm{~m}$ & 116,48 & 121,26 \\
\hline $\mathrm{OMe}$ & - & - & $3,96 s$ & - & 55,6 \\
\hline $\mathrm{OH}$ & $12,83 s$ & $12,09 s$ & $12,19 s$ & - & - \\
\hline
\end{tabular}

* valores podem estar trocados. 


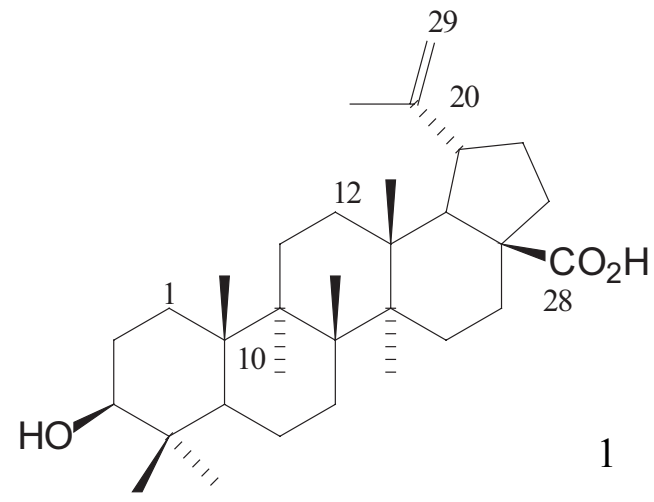<smiles></smiles><smiles>O=c1c(O)c(-c2ccc(O)c(O)c2)oc2cc(O)cc(O)c12</smiles><smiles>COc1ccc([C@@H]2Oc3cc(O)cc(O)c3C(=O)[C@H]2O)cc1O</smiles>

Figura 1. Substâncias isoladas de Davilla rugosa

na proporção de 9:1 e 4:1. Foram obtidas 36 frações de $15 \mathrm{~mL}$ cada e, as frações eluídas com $\mathrm{CHCl}_{3}: \mathrm{MeOH}$ (9:1) foram agrupadas e submetidas a permeção em gel de Sephadex LH-20 (Pharmacia) e eluídas com MeOH: $\mathrm{CH}_{2} \mathrm{Cl}_{2}$ (3:2). Desta maneira foram isolados quercetina (3, 5,3 mg) e 4'-O-metil taxifolina (4, 6,3 mg).

Ácido betulínico. Sólido amorfo branco. PF. 210$5{ }^{\circ} \mathrm{C}$. RMN de ${ }^{13} \mathrm{C}[75 \mathrm{MHz}$, piridina-d,$\delta(\mathrm{ppm})]$ : Dados de $\mathrm{RMN}$ de ${ }^{13} \mathrm{C}$ do ácido betulínico (1) e comparação com os descritos na literatura para o derivado metilado [75 $\mathrm{MHz}$, piridina-d $\left.\mathrm{d}_{5}, \delta(\mathrm{ppm})\right]: 39,5(\mathrm{C}-1) ; 28,1(\mathrm{C}-2) ; 78,6$ (C-3); 38,1 (C-4); 56,2 (C-5); 19,0 (C-6); 35,1(C-7); 39,9 (C-8); 51,2 (C-9); 38,0 (C-10); 21,4 (C-11); 26,4 (C12); 38,9 (C-13); 43,4 (C-14); 30,5 (C-15); 33,1(C-16); 57,2 (C-17); 50,0 (C-18); 48,0 (C-19); 151,8 (C-20); 31,4 (C-21); 37,8 (C-22); 28,9 (C-23); 16,8* (C-24); 16,7* (C-25); 16,6* (C-26); 15,2 (C-27); 179,7 (C-28); 110,3 (C-29); 19,8 (C-30). * Os sinais podem estar trocados. Identificação realizada através da comparação com dados descritos na literatura do derivado metilado (Wenkert et al., 1978).

\section{RESULTADOS E DISCUSSÃO}

As substâncias isoladas tiveram suas estruturas elucidadas através da análise dos dados de RMN. A friedelina e a sitostenona, isolados do extrato hexânico, foram identificados através da comparação direta dos dados espectrométricos registrados com os descritos na literatura (David et al., 2003; Correia et al., 2003). $\mathrm{O}$ espectro de $\mathrm{RMN}$ de ${ }^{13} \mathrm{C}$ de $\mathbf{1}$, mostrou sinais de 30 carbonos, característicos de triterpenos. A análise dos dados obtidos nos espectros de DEPT 135 e $90^{\circ}$ permitiram atribuir o sinal em $\delta 179,7$ à carbonila, característica de grupo ácido. Além disso, a presença de dois carbonos $s p^{2}$ $(\delta 109,3$ e 151,8$)$ e um oximetínico $(\delta$ 78.6) permitiram sugerir que a substância tratava-se de um triterpeno ácido de esqueleto lupano. A comparação dos dados obtidos (Tabela 1) com os descritos na literatura (Wenkert et al., 1978) permitiu concluir que este tratava-se do ácido betulínico.

A flavona narigenina (2) e a quercetina (3) foram identificadas através dos dados obtidos no RMN de ${ }^{1} \mathrm{H}$ e de ${ }^{13} \mathrm{C}$ (Tabela 2) e em comparação com os dados descritos na literatura (Markham; Geiger, 1994). Enquanto que, a estrutura do diidroflavanol 4 foi determinada como sendo a 4'-O-metil taxifolina através dos dados de RMN (Tabela 2) e por espectrofotometria no ultravioleta com adição de reagentes de deslocamento. Pelo espectro de $\mathrm{RMN}$ de ${ }^{1} \mathrm{H}$ e de ${ }^{13} \mathrm{C}$ foi possível determinar o esqueleto flavanoídico e seu padrão de oxigenação. No entanto, os dados não foram conclusivos sobre a posição do grupo metoxílico ( $\delta$ 3,8 no espectro de $\mathrm{RMN}$ de ${ }^{1} \mathrm{H}$ ). A comparação dos espectros no UV da substância $(\lambda=360 \mathrm{~nm})$ e com adição de solução de $\mathrm{AlCl}_{3}(\lambda=374 \mathrm{~nm})$ permitiu concluir que o grupo metoxílico encontrava-se no anel $\mathrm{B}$, devido ao deslocamento batocrômico $(\Delta \lambda=14 \mathrm{~nm})$ observado, seguido de deslocamento hipsocrômico quando houve 
destruição do complexo formado após adição de solução de $\mathrm{HCl}$. Entretanto o não aparecimento de deslocamento batocrômico após adição de solução de $\mathrm{NaOMe}$, na cubeta, foi indicativo de grupo metoxílico estava localizado no C-4' do anel B do sistema. A relação trans entre o grupo $\mathrm{OH}$ em $\mathrm{C}-3$ e o anel aromático em $\mathrm{C}-2$ pode ser obtida a partir da constante de acoplamento observada no espectro de $\mathrm{RMN}$ de ${ }^{1} \mathrm{H}$ entre $\mathrm{H}-3 / \mathrm{H}-2(\mathrm{~J}=10 \mathrm{~Hz})$, indicando que os dois prótons encontravam-se em pseudo-axial.

Embora o estudo fitoquímico de D. rugosa tenha sido parcialmente realizado, foram isoladas quatro substâncias todas com atividade biológicas comprovadas. Deve-se destacar que o ácido betulínico foi obtido em quantidade considerável $(0,4 \%$ peso seco) demonstrando que a espécie é uma fonte alternativa para esta substância.

\section{AGRADECIMENTOS}

Os autores agradecem o CNPq e a FAPESB pelas bolsas de Iniciação científica de JCS e de pesquisa dos autores como também pelos auxílios financeiros para desenvolvimento do trabalho.

\section{REFERÊNCIAS}

Bacchi EM 1986. Ação anti-úlcera e cicatrizante de algumas plantas brasileira. Rev Bras Farmacogn 1: 93-100.

Carlos IZ, Lopes FCM, Benzatti FP, Carli CBA, Marques FM, Jordão-Júnior CM, Rinaldo D, Calvo TR, Santos LC, Vilegas W 2005. Ação do extrato metanólico e etanólico de Davilla elliptica St. Hill. (Dilleniaceae) na resposta imune. Rev Bras Farmacogn 15: 4450.

Coimbra R 1942. Notas de Fitoterapia. 1a. Ed. Rio de Janeiro: Ed. Carlos da Silva Araújo S. A., p. 87.

Corrêa MP 1984. Diccionário das Plantas Úteis do Brasil. Vol. II. Ministério da Agricultura - Instituto Brasileiro de Desenvolvimento Florestal, p. 266.

Correia SJ, David JPL, David JM 2003. Constituintes das cascas de Tapirira guianensis (Anacardiaceae). Quim Nova 26: 36-38.

David JM, Cruz FG, Guedes MLS, Chavez JP 1996. Flavonol glycosides from Davilla flexuosa. J Braz Chem Soc 7: 115-118.

David JM, Santos FA, Guedes MLS, David JP 2003. Flavonóides e triterpenos de Stigmaphyllom paralias. Quim Nova 26: 484-487.

Guaraldo L, Chagas DA, Konno AC, Korn GP, Pfiffer T, Nasello AG 2000. Hydroalcoholic extract and fractions of Davilla rugosa Poiret: effects on spontaneous motor activity and elevated plus-maze behaviour. $J$ Ethnopharmacol 72: 61-67.

Guaraldo L, Sertie JA, Bacchi EM 2001. Antiulcer action of the hydroalcoholic extract and fractions of Davilla rugosa Poiret in the rat. J Ethnopharmacol 76: 191195.

Markham KR, Geiger H 1994. ${ }^{1} \mathrm{H}$ Magnetic nuclear resonance spectroscopy of flavonoids and their glycosides in hexadeuterodimethylsulfoxides. In: The Flavonoids:
Advances in research since 1986. Harbone, JB (Ed.). Great Britain: Chapman \& Hall, p. 441-473.

Michelin DC, Ilha SM, Rinaldo D, Sannomiya M, Santos LC, Vilegas W, Salgado HRN 2005. Antimicrobial activity of Davilla elliptica St. Hill (Dilleniaceae). Rev Bras Farmacogn 15: 209-211.

Schultz A 1984. Introdução a Botânica Sistemática. 4a. Ed. Porto Alegre: Editora Universidade Federal do Rio Grande do Sul, vol. 2, p. 121.

Soares ML, Rezende MH, Ferreira HD, Figueiredo ADL, Bustamante KGL, Bara MTF, Paula JR 2005. Caracterização farmacognóstica de folhas de Davilla elliptica St. Hil. (Dilleniaceae). Rev Bras Farmacogn 15: 352-360.

Wenkert E, Baddeley V, Burfett IR, Moreno LN 1978. C13 Nuclear magnetic-resonance spectroscopy of naturally-occurring substances. Triterpenes related to lupane and hopane. Org Magn Res 11: 337-343. 